\title{
A percepção do outro no ambiente virtual de aprendizagem: presença social e suas implicações para Educação a Distância
}

\author{
WILLYANS GARCIA COELHO \\ Instituto Federal de Pernambuco, Recife, PE, Brasil \\ PATRICIA CABRAL DE AZEVEDO RESTELLI TEDESCO \\ Universidade Federal de Pernambuco, Recife, PE, Brasil
}

\section{RESUMO}

O objetivo deste artigo foi delimitar o conceito de presença social e apontar suas implicações para a Educação a Distância (EAD). Presença social é um termo da Psicologia relacionado à percepção do indivíduo sobre a maneira como ele se sente na interação com os outros, na comunicação mediada por tecnologia. São apresentados estudos acerca dos desafios para promover a aprendizagem colaborativa e a interação em ambientes virtuais e, então, discutidos o papel da tecnologia e dos comportamentos das pessoas nesse processo. As reflexões indicam que estudar o nível de presença social nos ambientes virtuais de aprendizagem (AVA) é fundamental para compreender a interação entre os aprendizes e promover a aprendizagem colaborativa na EAD.

\section{PALAVRAS-CHAVE}

educação a distância; ambiente virtual de aprendizagem; presença social; interação; aprendizagem colaborativa. 


\title{
THE PERCEPTION OF THE OTHER IN THE VIRTUAL LEARNING ENVIRONMENT: SOCIAL PRESENCE AND ITS IMPLICATIONS FOR DISTANCE EDUCATION
}

\begin{abstract}
The aim of this paper was to establish the concept of social presence and to point out its implications for Distance Education. Social presence is a term originated from Psychology, and refers to the individual's perception about the way he or she feels when interacting with others using computer-mediated technology. We present studies that discuss the challenges of promoting collaborative learning and interaction in virtual environments; moreover, we analyze the role of technology and the learners' behavior in this process. Our findings indicate that studying the level of social presence in virtual learning environments (VLE) is crucial for understanding the interaction between learners, as well as for promoting collaborative learning in Distance Education.
\end{abstract}

\section{KEYWORDS}

distance education; virtual learning environment; social presence; interaction; collaborative learning.

\section{LA PERCEPCIÓN DE LA OTRA PERSONA EN EL ENTORNO VIRTUAL DE APRENDIZAJE: PRESENCIA SOCIAL Y SUS IMPLICACIONES PARA LA EDUCACIÓN A DISTANCIA}

\section{RESUMEN}

El propósito de este artículo es definir el concepto de presencia social y señalar sus repercusiones para Educación a Distancia. La presencia social es un término de la Psicología que se relaciona con la percepción del individuo de cómo se siente en la interacción con los demás en la comunicación mediada por la tecnología. Estaremos presentando estudios que discutirán los desafíos para promover el aprendizaje colaborativo y la interacción en entornos virtuales y entonces, aprendizaje colaborativo. Los resultados del estudio indican que estudiar el nivel de presencia social en los entornos virtuales de aprendizaje (EVA) se vuelve crítico para comprender la interacción entre los aprendices y fomentar el aprendizaje colaborativo en la Educación a Distancia.

\section{PALABRAS CLAVE}

educación a distancia; entorno virtual de aprendizaje; presencia social; interacción; aprendizaje colaborativo. 


\section{INTRODUÇÃO}

Pesquisas recentes têm apontado a importância da interação social e do senso de pertencimento como elementos-chave para a aprendizagem e para a construção colaborativa do conhecimento em ambientes virtuais (Onrubia, Colomina e Engel, 2010). Contudo, percebe-se que na comunicação mediada por tecnologia há uma perda significativa da linguagem corporal subjacente ao ato de interagir, como o olhar, a expressão facial, a postura, os gestos, entre outros elementos. Diante das diferenças entre a interação face a face e a interação mediada por tecnologia, pesquisadores têm defendido a importância de estudos acerca da promoção de espaços de interação virtual que facilitem a aprendizagem colaborativa (Boling et al.,2012; Hill, Song e West, 2009).

É nesse contexto que ganha relevância o conceito de presença social. Considerado um dos conceitos mais populares para descrever e compreender o modo como as pessoas interagem em ambientes virtuais (Lowenthal, 2010), trata-se de um termo da Psicologia relacionado à percepção do indivíduo sobre a maneira como ele se sente na interação com os outros (Kim, 2011). Esse é um dos principais desafios para a aprendizagem colaborativa por meio da internet e vem sendo estudado por diversos pesquisadores (Deschryver et al., 2009; Gunawardena e Zittle, 1997; Jin, 2009; Kehrwald, 2008; Kim, 2011; Lin, 2004; Lowenthal, 2010; Russo e Benson, 2005; Tu, 2002).

Diante dessa perspectiva, o objetivo do presente trabalho foi refletir sobre o conceito de presença social e suas implicações para a Educação a Distância (EAD), em especial analisar o seu papel para o surgimento da interação no ambiente virtual e discutir suas potencialidades para promover a aprendizagem colaborativa.

\section{APRENDIZAGEM COLABORATIVA NA INTERNET}

A oferta de atividades para aprendizagem por meio da web é muito diversificada, incluindo desde materiais de autoinstrução até videoconferências em tempo real. Ou seja, desde atividades sem qualquer interação entre as pessoas até aquelas que exigem interação constante. Outro aspecto importante está relacionado ao tempo. Há atividades assíncronas, em que cada pessoa acessa o conteúdo no momento que desejar, e atividades síncronas, que demandam a participação de todos os aprendizes no mesmo ambiente e ao mesmo tempo.

Enfim, a tecnologia pode ser utilizada tanto em uma abordagem tradicional de transmissão mecanicista de conteúdos, isto é, em uma simulação de aulas presenciais, quanto em uma abordagem inovadora, a partir da exploração das características da própria tecnologia disponível, com base na interação e na produção colaborativa de conhecimento (Almeida, 2003). Nesse momento, dispomos de diversos recursos para experimentar novas formas de disponibilizar conteúdos, bem como para desenvolver novas estratégias educacionais (Abbad, Carvalho e Zerbini, 2006).

$\mathrm{O}$ ambiente virtual de aprendizagem (AVA) é a parte mais visível de todo o arcabouço tecnológico utilizado para dar suporte à realização da EAD. É por meio dele que são disponibilizados os conteúdos dos cursos e são promovidas as interações entre os participantes. 
Existem muitas formas de conceituar um AVA, as quais têm mudado ao longo do tempo (Mueller e Strohmeier, 2010). Porém, muitas tentativas acabam por definir processos, apresentar ferramentas ou enumerar requisitos mínimos, confundindo conceituação com descrição. E mais, deixam transparecer nessa tentativa de definição uma ou outra concepção sobre educação, privilegiando determinada prática educacional em detrimento das demais. Numa visão mais conciliadora, Araújo Junior e Marquesi (2009) propõem uma definição operacional, a partir da perspectiva do usuário, na qual o AVA seria um ambiente que utiliza a tecnologia da internet para simular o ambiente presencial de aprendizagem.

Essa definição nos dá uma perspectiva mais ampla, resguardando possíveis diferenças entre os ambientes virtuais quanto aos seus recursos, formatos, modos de utilização e objetivos. $\mathrm{Na}$ realidade, as práticas pedagógicas são mediadas pelos diferentes recursos disponíveis no ambiente, e a avaliação acerca da utilização desses recursos deve estar alinhada à concepção educacional (Behar et al., 2007). Por isso, no processo de escolha do AVA, devem-se analisar as implicações técnicas e pedagógicas envolvidas, bem como ter os objetivos claros, a fim de garantir que a escolha atenda às reais necessidades, para dar maior credibilidade às ações de aprendizagem propostas por meio da EAD (Mehlecke, 2006).

Em função dos objetivos propostos e da concepção pedagógica subjacente à escolha ou à própria construção e utilização do AVA, é possível distinguir ao menos dois tipos de abordagens mais comuns na EAD: uma apoiada pela proposta de autoinstrução e independência dos aprendizes; outra, pela concepção de construção colaborativa do conhecimento, por meio da interação. Para Primo (2003), um AVA pode servir para ambas as abordagens, ou seja, tanto é possível promover uma aprendizagem colaborativa a partir do diálogo quanto é possível distribuir “apostilas digitais" entre alunos anônimos. $O$ fato é que as duas abordagens não são excludentes; pelo contrário, um dos desafios da EAD é encontrar o equilíbrio entre o estudo independente e as atividades colaborativas (Mattar, 2009).

Os estudos independentes (ou autossuficientes) - por meio dos quais os aprendizes acessam os conteúdos no ambiente virtual e realizam suas atividades isoladamente, sem interação com os demais aprendizes - vêm dominando as iniciativas de $\mathrm{EAD}$ desde os seus primórdios, ganhando força recentemente com a atratividade e a disseminação dos recursos multimídia (Barberà e Rochera, 2010). Contudo, com o avanço das ferramentas colaborativas, a promoção da colaboração em ambientes virtuais tem sido considerada como aquela que atende mais adequadamente aos novos paradigmas que enfatizam a aprendizagem colaborativa (Capdeferro e Romero, 2012; Salvat e Silva, 2006).

Para compreender essa relação entre colaboração e tecnologia, emerge um novo campo de estudo nas ciências da educação: a Aprendizagem Colaborativa Apoiada por Computador - CSCL (Computer-Supported Collaborative Learning). Nessa área, apesar das diferenças existentes, costuma-se entender a aprendizagem colaborativa como "um processo de interação no qual se compartilham, negociam-se e constroem-se significados conjuntamente para solucionar um problema, criar ou produzir algo" (Onrubia, Colomina e Engel, 2010, p. 211). 
O interesse atual pelo processo de aprendizagem colaborativa na educação tem se dado em função de diversos fatores. Colomina e Onrubia (2004) apontam dois aspectos de maior destaque: a compreensão de que a aprendizagem é um processo social, especialmente pelas concepções da teoria sociocultural de Vygotsky; e o crescente número de pesquisas que demonstram a maior efetividade, do ponto de vista acadêmico, das atividades colaborativas em sala de aula. Consolidando os resultados encontrados em diversos estudos, Oncu e Cakir (2011) concluem que a colaboração online está associada ao aumento no volume e na qualidade do envolvimento dos aprendizes, na sua satisfação, no seu engajamento e na sua habilidade de pensar criticamente.

Ao ser definida como um processo interativo, a aprendizagem colaborativa depende da interação entre as pessoas no ambiente virtual. Congruentes com essa perspectiva, a interação social e o senso de pertencimento no AVA são considerados fatores essenciais para a construção colaborativa do conhecimento. A partir disso, conclui-se que, se houve colaboração, houve interação; e que, se não houver interação, não haverá colaboração (Kreijns, Kirschner e Jochems, 2003; Onrubia, Columina e Engel, 2010).

A interação é considerada, hoje, um conceito polissêmico em função do seu uso nas mais diversas áreas, da Educação à Computação, da Psicologia ao Marketing. Contudo, numa concepção relacionada à EAD, costuma-se considerar a interação como uma troca ativa de ações e informações entre as pessoas (Borokhovski et al., 2012).

Nessa concepção, Woo e Reeves (2007) consideram que manter a interação no AVA é um desafio muito maior do que fazê-lo em contextos presenciais, especialmente por conta da separação, no tempo e no espaço, provocada pelo uso da tecnologia. Eles argumentam que a aprendizagem colaborativa não ocorre com qualquer tipo de interação, e sim com a realização daquilo que chamam de "interação significativa”. Esta deve estimular a curiosidade intelectual das pessoas, envolvê-las em atividades produtivas de ensino e influenciar diretamente sua aprendizagem. Para tanto, a interação entre as pessoas no AVA deveria incluir ações de responder, negociar (interna e socialmente), argumentar pontos de vista, agregar novas ideias e oferecer novas perspectivas a discussões de problemas reais.

$\mathrm{O}$ AVA é reconhecido como um meio adequado à realização dessas ações de interação. Contudo, sua utilização para esse fim requer a compreensão das suas limitações e a aplicação de regras específicas, por meio da adoção de modelos que atendam às singularidades de cada situação (Morueta, Gómez e Gómez, 2011).

$O$ fato é que se torna um grande desafio promover interação significativa entre as pessoas utilizando somente ferramentas de comunicação assíncrona, baseadas exclusivamente em texto, tão comuns em diversas práticas de EAD. Para Onrubia, Colomina e Engel (2010), tal forma de comunicação impõe uma série de restrições "que podem produzir certa sensação de solidão nas pessoas e diminuir sua capacidade de estabelecer relações interpessoais, dificultando a formação do diálogo aberto que apoie e promova a troca crítica e construtiva de ideias".

Hill, Song e West (2009) apontam que essa dificuldade para gerar interação no AVA estaria relacionada a um dos maiores desafios da comunicação mediada por tecnologia: a falta de pistas sociais que facilitem a interação entre as pessoas, como a expressão facial, a linguagem corporal e o tom de voz. 
Diante dessa realidade, mesmo com o avanço das ferramentas tecnológicas e com a realização das mais diversas experiências em EAD, para Boling et al. (2012) há, ainda hoje, muito o que pesquisar para criar ambientes virtuais altamente interativos e que, de fato, favoreçam a promoção da aprendizagem colaborativa. Oncu e Cakir (2011) defendem que a melhoria do engajamento e da colaboração dos aprendizes deve ser uma das prioridades nas pesquisas futuras sobre AVA.

Portanto, se para promover a aprendizagem colaborativa é essencial que ocorra a interação entre as pessoas, e, por sua vez, a interação tem sido dificultada pela falta de pistas sociais na comunicação mediada pelo computador, emerge aqui uma importante área de estudo.

\section{PRESENÇA SOCIAL NA EDUCAÇÃO A DISTÂNCIA}

O ser humano é essencialmente social. Temos a capacidade de nos relacionar com o outro desde o nascimento (Wallon, 1995). Por isso, já no início da vida, nos organizamos em grupos familiares que apoiam o desenvolvimento de cada pessoa. E isso se estende por todas as nossas atividades sociais, seja na família, seja na escola, no trabalho ou no lazer. Em todos esses momentos, sempre necessitamos estar com o outro.

Uma de nossas capacidades que mais se apoiam nesses relacionamentos sociais é a de aprender. Para Vygotsky (1991), a construção do conhecimento é uma atividade essencialmente social. Nessa perspectiva sociocultural, "os processos de aprendizagem fundamentam-se na ideia de que as atividades humanas estão posicionadas em contextos culturais e são mediadas pela linguagem e por outros sistemas simbólicos" (Cubero e Luque, 2004, p. 105).

Dessa forma, a educação não pode se resumir a uma simples transferência de conteúdo formal. Há, nesse processo, um caráter socializante que envolve emoção, sensibilidade e afetividade (Freire, 1998). Nesse contexto, a construção de conhecimento ocorre por meio dos relacionamentos entre as pessoas, da interação de uns com os outros em ambientes de aprendizagem, em um processo dialógico (Freire, 1987).

De forma geral, quando se aborda a necessidade de interação social para a aprendizagem, o que visualizamos e temos como modelo, inicialmente, é o relacionamento face a face, o estar fisicamente no mesmo ambiente que a outra pessoa. Nessa interação face a face, muitos fatores influenciam a intensidade e a qualidade do relacionamento estabelecido. Além da linguagem verbal, Birdwhistell (1970) demonstra que há uma linguagem corporal subjacente ao ato de falar, expressada por meio do olhar, da expressão facial, da postura e dos gestos. Assim como a fala, tais sinais não verbais são adquiridos no desenvolvimento do sujeito, por meio da interação social, e compartilhados por determinada cultura.

A partir dessa constatação, Gumperz (2002) analisa que a comunicação humana é delimitada por um conjunto de sinais verbais e não verbais construídos culturalmente e internalizados pelas pessoas ao longo do seu desenvolvimento. Tais sinais seriam coordenados de forma a constituir pistas de contextualização, transmitindo com maior clareza aquilo que se deseja expressar na comunicação face a face. Portanto, a riqueza da comunicação oral não está apenas no que se fala, mas, especialmente, na forma como se fala. 
Analisando especificamente a função do olhar na comunicação face a face como acontecimento único para estabelecer a ligação entre as pessoas, Quintas-Mendes, Morgado e Amante (2010) afirmam que "aparentemente tudo conspira contra a comunicação a distância”. Afinal, o ser humano nasce com diversos recursos para manter relações interpessoais próximas e intensas, mas não para promover o relacionamento na comunicação por meio da tecnologia.

Dessa forma, quando a interação deixa de ser face a face e passa a ser mediada por algum aparato tecnológico, sejam as correspondências, o telefone ou, mais recentemente, o computador, alguns desses sinais verbais ou não verbais são perdidos em maior ou menor grau. Mesmo assim, ainda é possível estabelecer a interação entre as pessoas. Essa capacidade de perceber a relevância do outro na interação denominamos presença social.

\section{CONCEITO DE PRESENÇA SOCIAL}

Short, Williams e Christie (1976) têm sido apontados como os primeiros a definir o conceito de presença social, comparando as interações mediadas por tecnologia (por exemplo, correspondência, telefone, entre outras) com as interações presenciais (Kehrwald, 2008). Considerando o relacionamento face a face como tendo o grau máximo de presença social e a correspondência, o grau mínimo, eles investigaram em que posição desse contínuo estaria situada a interação por telefone. A partir desse estudo, Short, Williams e Christie (1976) definiram a presença social como o grau de relevância da outra pessoa na interação e a consequente relevância do relacionamento interpessoal, ou seja, nossa capacidade de perceber a significância do outro na interação.

Como qualquer fenômeno psicossocial, a abordagem ao conceito de presença social mostra pequenas diferenças de perspectiva entre os diversos pesquisadores. Lowenthal (2010) aponta três fases distintas da evolução e do uso desse conceito. A primeira fase, na década de 1970, teria como foco os meios de telecomunicação. Inicialmente, as pesquisas consideravam o fenômeno de presença social exclusivamente como um atributo do meio estudado, concentrando o foco na capacidade do meio de comunicação em transmitir as informações necessárias para tornar a percepção da interação como sendo real (Kehrwald, 2008).

Na segunda fase, iniciada na década de 1980, com a difusão dos computadores pessoais, o foco das pesquisas de presença social passou a ser a comunicação mediada pelo computador. Com os inúmeros recursos que o computador oferecia, as pesquisas buscaram explorar, além da tecnologia utilizada, as estratégias empregadas pelas pessoas para transmitir emoções por meio do computador e, com isso, estabelecer uma interligação entre elas, como os emoticons ${ }^{1}$. Não se observa mais apenas as características do meio, mas, especialmente, a forma como as pessoas

1 Emoticon-Derivada da junção dos termos em inglês emotion (emoção) + icon (ícone), trata-se de uma forma de comunicação paralinguística utilizada para transmitir o estado emotivo da pessoa por meio de uma sequência de caracteres tipográficos ou uma pequena imagem. Exemplos:^^^ :-) :-(; :) : 
utilizam os recursos oferecidos por esses meios para se fazer perceber. Dessa forma, o sujeito deixa de ter papel passivo e assume papel ativo junto ao meio utilizado.

$\mathrm{Na}$ terceira fase, que vem desde meados da década de 1990 até os dias de hoje, o conceito de presença social começou a ser explorado mais fortemente na área de EAD, especialmente nos AVA. Nesse terceiro momento, são investigadas as percepções das pessoas e não apenas as características do meio de comunicação. Dessa forma, a presença social envolve três aspectos: os recursos que o meio oferece, as estratégias que os participantes utilizam para se projetar como pessoas por meio desses recursos, e a forma como se dá a percepção do outro nesses ambientes.

Nessa perspectiva de considerar o meio, o indivíduo e sua relação com o outro, $\mathrm{Tu}$ (2002) distinguiu pelo menos três dimensões do conceito de presença social: o contexto social, a comunicação online e a interatividade. $O$ contexto social compreende as características dos usuários e sua percepção acerca do ambiente virtual, como a confiança e a disponibilidade do ambiente, os propósitos de aprendizagem e a língua utilizada. Já a dimensão da comunicação online está relacionada à habilidade da pessoa em utilizar o computador, empregar uma linguagem apropriada à rede e escrever e interpretar textos. Por último, a interatividade inclui os estilos de comunicação da pessoa, o tempo de resposta às mensagens, o feedback às mensagens dos outros e suas estratégias de aprendizagem.

Portanto, a concepção de presença social foi desenvolvida no contexto das telecomunicações, influenciando as pesquisas sobre comunicação mediada por computador e, atualmente, sendo explorada, sobretudo, nas pesquisas da área de EAD. Em cada fase, uma nova perspectiva foi considerada. Inicialmente, apenas as características do meio tecnológico, depois, as estratégias das pessoas na utilização do meio tecnológico e, por fim, a maneira como ocorre a percepção do outro nesse ambiente virtual.

Dessa forma, o conceito de presença social evoluiu no sentido de considerar a qualidade dos relacionamentos estabelecidos entre as pessoas, em vez de considerar apenas o sujeito como ente passivo diante do meio tecnológico utilizado. Se apenas o meio fosse determinante para a presença social, todas as pessoas teriam a mesma percepção quando mediadas pelo mesmo recurso tecnológico. Mas esse não é o caso,já que pessoas distintas têm níveis de presença social distintos (Gunawardena, 1995; Tu, 2002).

Consolidando essa evolução do conceito, Kim (2011) define presença social como a percepção do indivíduo sobre o grau de proximidade e afiliação estabelecido com os outros participantes de um ambiente de interação mediado por tecnologia, refletindo a dinâmica da relação entre as pessoas. Nessa perspectiva, tal percepção seria influenciada tanto pelo meio tecnológico utilizado para promover a interação quanto pelas características pessoais de cada indivíduo. É essa a definição adotada no presente trabalho.

\section{PRESENÇA SOCIAL E SUAS IMPLICAÇÕES NA EDUCAÇÃO A DISTÂNCIA}

$O$ conceito de presença social ganhou relevância na EAD especialmente por conta das correlações positivas encontradas pelos pesquisadores da área. Inúmeros estudos têm se dedicado a demonstrar que o grau de presença social percebido em ambientes virtuais pode ser correlacionado a aspectos essenciais para a EAD, como a evasão dos cursos, a avaliação da aprendizagem, a satisfação com o curso, entre outros fatores, que serão descritos a seguir. 
Uma das primeiras descobertas é a forte correlação entre a presença social e o grau de satisfação dos aprendizes em participar de uma conferência mediada por computador (Gunawardena e Zittle, 1997). Por meio da análise fatorial de um questionário aplicado aos participantes de uma conferência online sobre EAD, os pesquisadores identificaram três fatores relacionados à satisfação dos participantes, sendo a presença social o fator de maior impacto, contribuindo com $60 \%$ da variância, seguido por percepção de oportunidades iguais (equidade), com 7\%, e, por fim, habilidades técnicas, com apenas $4 \%$. Dessa forma, os pesquisadores concluíram que o nível de presença social é, isoladamente, um forte preditor do grau de satisfação dos estudantes. Assim, quanto maior o nível de presença social percebido pelos participantes, maior a sua satisfação com o evento.

Outros estudos apontam alta correlação entre a presença social e a aprendizagem percebida pelos aprendizes, bem como entre a presença social e a satisfação com o tutor/instrutor (Richardson e Swan, 2003; Russo e Benson, 2005). Pessoas com alta percepção de presença social também sentiram que sua aprendizagem foi maior do que a daqueles que tinham baixa percepção de presença social. No mesmo estudo, também foi verificado que aprendizes com alto grau de presença social apresentaram-se muito mais satisfeitos com os seus tutores, tanto em relação à quantidade quanto em relação à qualidade da interação.

A retenção dos aprendizes nos cursos também está correlacionada com a presença social (Liu, Gomez e Yen, 2009). Quanto maior o grau de presença social percebida pela pessoa, menor a chance de evasão do curso. Essa mesma pesquisa demonstrou ainda que o grau de presença social percebido pelo aprendiz também está correlacionado à sua avaliação ao final no curso. Assim, quanto maior a presença social, maior a nota do aprendiz.

A presença social é um fator-chave para a percepção da qualidade na $\mathrm{EAD}$ a partir da perspectiva do aluno (Cobb, 2009). A criação de um alto grau de presença social pode promover estratégias mais parecidas com aquelas dos cursos presenciais e, dessa forma, tornar o AVA mais familiar aos estudantes (Mackey e Freyberg, 2010). Os pesquisadores sugerem que a implantação de estratégias para aumentar a presença social pode criar um clima de aprendizagem mais positivo e, assim, melhorar a experiência global de aprendizagem, ao tornar o ambiente virtual mais agradável para os alunos.

Os resultados dessas pesquisas apontam para a importância de aumentar e manter um elevado nível de percepção da presença social no ambiente virtual. Quanto maior o nível de presença social, maior a interação entre alunos e tutores, melhor a percepção de aprendizagem, maior a familiaridade com o ambiente virtual, menor a evasão, melhor a avaliação final do aluno e, sobretudo, maior o nível de satisfação dos estudantes com os cursos.

\section{PRESENÇA SOCIAL, INTERAÇÃO E COLABORAÇÃO NO AMBIENTE VIRTUAL}

O principal aspecto relacionado à presença social é sua contribuição significativa para fomentar o potencial de interação nos AVA (Gunawardena, 1995; Tu, 2000). As pessoas precisam sentir alguma forma de ligação com as outras para que, de fato, compartilhem ideias e opiniões (Kear,2010). Por isso, muitos pesquisadores 
têm se interessado em estudar formas de aumentar o nível de presença social dos aprendizes (Sung e Mayer, 2012).

Há certas crenças que têm sido limitantes nesse processo: primeiro, acreditar que basta colocar os estudantes em grupo para que a interação surja; segundo, a ideia de que a interação acontece como simples decorrência da disponibilidade de recursos tecnológicos para tal; e terceiro, a tendência de limitar a interação dos aprendizes aos conteúdos de aprendizagem (Kreijns, Kirschner e Jochems, 2003).

Para que haja interação, cada indivíduo deve reconhecer a presença dos demais no AVA. Quanto maior a interação entre os alunos, maior o estímulo e maior a motivação para se engajarem no processo de aprendizagem colaborativa (Tu, 2000). Por outro lado, se a presença social for baixa, a interação não ocorrerá, o que afetaria fortemente a socialização da aprendizagem num ambiente virtual. Entretanto, não é somente a quantidade ou a frequência das interações no AVA que aumentarão com a maior percepção de presença social, mas também a qualidade dessas interações (Tu e Mcisaac, 2002).

Portanto, para que a aprendizagem colaborativa ocorra, é essencial que haja interação. E para que haja interação, é imprescindível reconhecer a existência e a importância da outra pessoa no AVA. Na linha inversa, quanto maior o nível de presença social dos aprendizes, maior o potencial de interação, o qual, por sua vez, facilitará a ocorrência de aprendizagem colaborativa.

Importante observar que não se trata de causalidade, mas de potencialidade. Um alto nível de presença social não vai gerar, automaticamente, interação. Mas um baixo nível certamente limitará a interação de qualidade. Ou seja, a presença social é um pré-requisito para a interação, mas não é garantia. Existem outros fatores que influenciam tal relação. Da mesma forma, deve-se pensar na relação entre interação e colaboração. Para uma aprendizagem colaborativa, é essencial a existência de interação entre os aprendizes. Mas a interação não vai gerar, necessariamente, colaboração entre eles.

Diante dessas implicações da presença social para a interação e a colaboração, pesquisadores sugerem duas ações essenciais aos gestores da EAD: primeiro, a aferição inicial do grau de presença social dos aprendizes por um instrumento de medida validado, de modo a evitar os riscos associados aos casos de baixo grau de presença social; e segundo, uma intervenção efetiva com a utilização de estratégias para elevar o grau de presença social (Liu, Gomez e Yen, 2009). Mas que estratégias seriam essas? Quais os aspectos que influenciariam o nível de percepção de presença social dos aprendizes na EAD?

$\mathrm{Na}$ realidade, o nível de presença social no ambiente virtual pode ser influenciado tanto pelas características pessoais de cada um, quanto pelos recursos que o meio oferece para transmitir informações sociais e emocionais a respeito do outro (Kear, 2010; Sung e Mayer, 2012; Tu, 2000). A presença social decorre de uma inter-relação entre aquilo que o AVA oferece como recursos tecnológicos de comunicação e as características de cada pessoa que participa desse ambiente. Ou seja, para facilitar o aumento do nível de presença social torna-se essencial observar tanto os comportamentos que instrutores e aprendizes adotam no AVA quanto os recursos oferecidos pelo ambiente (Gunawardena, 1995). 
Quanto aos comportamentos que os instrutores e aprendizes deveriam adotar no AVA, Sung e Mayer (2012) sintetizaram as recomendações de diversos estudos em cinco pontos:

1. Expressar respeito pelos esforços das pessoas nas atividades de aprendizagem.

2. Compartilhar informações pessoais para construir relações sociais.

3. Promover um ambiente aberto e hospitaleiro para dar e receber feedback.

4. Ter consciência da identidade de cada pessoa, dirigindo-se a ela pelo nome.

5. Partilhar suas histórias e suas experiências pessoais.

Ainda em relação às características individuais, existem aspectos cuja influência é mais complexa. Pesquisadores têm enfatizado os efeitos das diferenças individuais ou grupais, relacionando-os às questões de gênero (Johnson, 2011; Kim, Kwona e Cho, 2011) e de percepção de privacidade (Tu, 2002), entre outros. Esses aspectos não podem ser manipulados, mas suas influências precisam ser compreendidas e consideradas.

Já os recursos tecnológicos podem ser mais facilmente manipulados e oferecidos no ambiente virtual. A cada dia são lançadas diversas ferramentas e criadas ideias que podem ser utilizadas isoladamente ou incorporadas ao AVA. Nesse sentido, pesquisas recentes têm sido feitas para avaliar o nível de presença social utilizando recursos específicos, como Facebook (Deschryver et al., 2009), Twitter (Dunlap e Lowenthal, 2009), YouTube (Borup, West e Graham, 2012) e mundos virtuais em 3D como o Second Life (Jin, 2009).

Numa tentativa de síntese dos aspectos que influenciam ou são influenciados pela presença social, a Figura 1 traz um panorama das inter-relações estabelecidas entre esses fatores.

Numa análise circunscrita ao processo, observa-se que a presença social é influenciada tanto pelas características individuais quanto pelos recursos oferecidos pelo ambiente. Por sua vez, o nível de presença social influencia a interação e, consequentemente, a colaboração. É dessa forma que o conceito de presença social se posiciona no contexto da $\mathrm{EAD}$, num processo de mediação entre características do meio e resultados do processo.

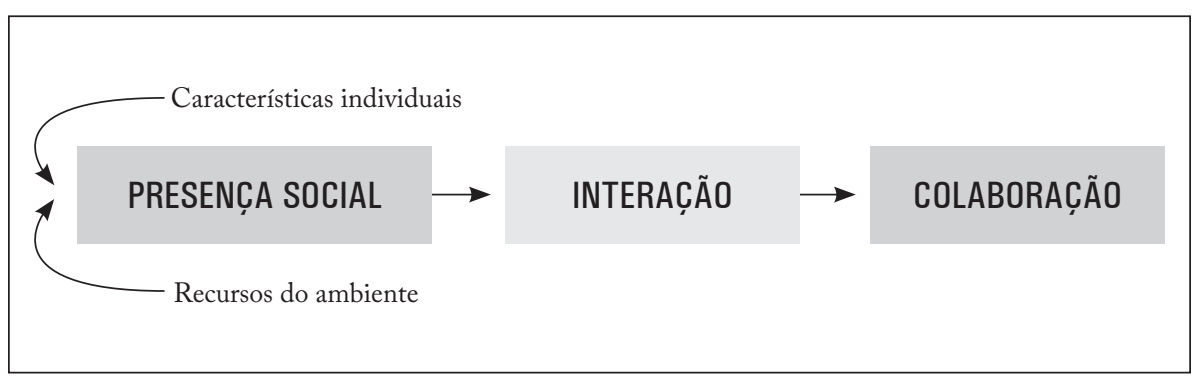

Figura 1 - O conceito de presença social no contexto da Educação a Distância [desenvolvido pelos autores]. 
É certo que na inter-relação entre os aspectos apresentados na Figura 1 há uma infinidade de variáveis intervenientes. Numa perspectiva mais ampla, ao compreender que o processo de aprendizagem é social (Vygotsky, 1991), convém salientar que todos os aspectos relacionados à presença social são mediados pela cultura. Portanto, a partir de uma perspectiva dialética, a cultura estabelecida em determinado grupo social certamente influenciará os aspectos apresentados na Figura 1, assim como esses aspectos influenciarão o meio cultural no qual estão inseridos. Nesse sentido, diversos pesquisadores têm relacionado os resultados dos seus estudos às influências culturais (Hall e Herrington, 2010; Kim, 2011; Yen e Tu, 2011).

Numa perspectiva mais específica a cada iniciativa de EAD, é importante destacar a influência das estratégias pedagógicas adotadas no AVA, como os objetivos traçados, as formas de distribuição do conteúdo, as atividades de aprendizagem propostas, o relacionamento entre as pessoas, as práticas para estimular o engajamento dos aprendizes, entre outros aspectos (Aragon, 2003; Wei, Chen e Kinshuk, 2012).

Diante desse cenário, pode-se concluir que a presença social se apresenta como um processo multidimensional, que envolve as pessoas nos níveis social (cultura) e individual (características pessoais), e o planejamento da ação educacional, nos níveis estratégico (desenho pedagógico do curso) e operacional (recursos tecnológicos) da EAD.

\section{CONSIDERAÇÕES FINAIS}

Os estudos individuais dominaram a EAD ao longo da sua evolução secular, por meio de recursos como apostilas por correspondência e programas de rádio ou TV que não favoreciam a comunicação dialógica entre os aprendizes. Mas, no contexto atual da EAD, os recursos tecnológicos da internet oferecem aos educadores novas oportunidades para promover uma abordagem educacional baseada na aprendizagem colaborativa entre aprendizes no AVA. Porém, apesar de todos os recursos tecnológicos disponíveis, observa-se que ainda hoje não se alcançou um alto grau de interatividade na EAD. Ou seja, não basta oferecer recursos, é preciso direcionar o olhar para as pessoas.

Nesse cenário, o conceito de presença social demonstra-se um importante fator para compreender as interações estabelecidas entre os aprendizes no AVA e suas repercussões, para que se faça emergir da interação uma aprendizagem colaborativa na EAD. Como apresentado anteriormente, as pesquisas sobre o tema revelam que elevar o grau de percepção da presença social dos aprendizes no AVA traz benefícios a diversos aspectos educacionais, como a interação e a colaboração entre os aprendizes, o nível de satisfação com o curso e com os tutores, entre outros. Mas, sobretudo, traz a oportunidade de oferecer um ambiente de aprendizagem humanizado e dialógico, mais próximo da nossa realidade social, por meio do qual as pessoas tenham a oportunidade de compartilhar e construir seu conhecimento.

Compreendida como multidimensional, a presença social se apresenta como um fenômeno que pode ser estudado em suas diversas dimensões, com metodologias e abordagens de pesquisa distintas e, especialmente, de forma multidisciplinar. 


\section{REFERÊNCIAS}

Abbad, G.; Carvalho, R. S.; Zerbini, T. Evasão em curso via Internet: explorando variáveis explicativas. RAE-eletrônica, v. 5, n. 2, jul./dez. 2006.

Almeida, M. E. B. D. Educação a distância na internet: abordagens e contribuições dos ambientes digitais de aprendizagem. Educação e Pesquisa, São Paulo, v. 29, n. 2, p. 327-340, jul./dez. 2003.

Aragon, S. R. Creating Social Presence in Online Environments. Nerw Directions for Adult and Continuing Education, v. 100, p. 57-68, jan./mar. 2003.

Araújo Junior, C. F. D.; Marquesi, S. C. Atividades em ambientes virtuais de aprendizagem: parâmetros de qualidade. In: Litto, F. M.; Formiga, M. (Orgs.). Educação a distância: o estado da arte. São Paulo: Pearson Education do Brasil, 2009.

BarberÀ, E.; Rochera, M. J. Os ambientes virtuais de aprendizagem baseados no projeto de materiais autossuficientes e na aprendizagem autodirigida. In: Coll, C.; Monereo, C. (Orgs.). Psicologia da educação virtual: aprender e ensinar com as tecnologias da informação e da comunicação. Porto Alegre: Artmed, 2010.

BEHAR,P.A. et al. Avaliação de ambientes virtuais de aprendizagem: o caso do ROODA na UFRGS. Revista Avances en Sistemas e Informática, Medellín, v. 4, n. 1, p. 87-99, jun. 2007. Birdwhistell, R. L. Kinesics and contest. Philadelphia: Pennsylvania Press, 1970.

Boling, E. C. et al. Cutting the distance in distance education: perspectives on what promote positive, online learning experience. Internet and Higher Education, v. 15, n. 2, p. 118-126, 2012.

Вовокноуккi, E. et al. Are contextual and designed student-student interaction treatments equally effective in distance education? Distance Education, v. 33, n. 3, p. 311-329, Nov. 2012.

Borup, J.; West, R. E.; Graham, C. R. Improving online social presence through asynchronous video. Internet and Higher Education, v. 15, p. 195-203, 2012.

Capdeferro, N.; Romero, M. Are online learners frustrated with collaborative learning experiences? The International Review of Research in Open and Distance Learning, v. 13, n. 2, p. 26-44, abr. 2012.

Совв, S. C. Social presence and online learning: a current view from a research perspective. Journal of Interactive Online Learning, v. 8, n. 3, p. 241-254, jan./mar. 2009.

Colomina, R.; Onrubia, J. Interação educacional e aprendizagem escolar: a interação entre os alunos. In: Coll, C.; Marchesi, Á.; Palacios, J. (Orgs.). Desenvolvimento psicológico e educação. 2. ed. Porto Alegre: Artmed, v. 2, 2004.

Cubero, R.; Luque, A. Desenvolvimento, educação e educação escolar: a teoria sociocultural do desenvolvimento e da aprendizagem. In: Coll, C.; Marchesi, Á.; Palacios, J. (Orgs.). Desenvolvimento psicológico e educação. 2. ed. Porto Alegre: Artmed, v. 2, 2004.

Deschryver, M. et al. Moodle vs Facebook: does using Facebook discussions in an online course enhance perceived social presence and student interaction? In: Proceedings of Society for Information Technology \& Teacher Education International Conference 2009. Chesapeake, VA: AACE, 2, mar. 2009. p. 329-336. 
Dunlap, J. C.; Lowenthal, P. R. Tweeting the night away: using Twitter to enhance social presence. Journal of Information Systems Education, v. 20, n. 2, p. 129-135, 2009. Freire, P. Pedagogia do oprimido. 17. ed. Rio de Janeiro: Paz e Terra, 1987.

. Pedagogia da autonomia: saberes necessários à prática educativa. 8. ed. Rio de Janeiro: Paz e Terra, 1998.

Gumperz, J. J. Convenções de contextualização. In: Ribeiro, B. T.; Garcez, P. M. Sociolinguística Interacional. São Paulo: Edições Loyola, 2002.

Gunawardena, C. N. Social Presence Theory and implications for interaction and collaborative learning in computer conferences. International Journal of Educational Telecommunications, v. 1, n. 2/3, p. 147-166, 1995.

Gunawardena, C. N.; Zittle, F. J. Social presence as a predictor of satisfaction within a computer-mediated conferencing environment. American Journal of Distance Education, v. 11, n. 3, p. 8-26, 1997.

Hall, A.; Herrington, J. The development of social presence in online Arabic learning communities. Australasian Journal of Educational Technology, v. 26, n. 7, p. 1012-1027, 2010. Hill, J. R.; Song, L.; West, R. E. Social learning theory and web-based learning enviroments: a review of research and discussion of implications. American Journal of Distance Education, v. 23, n. 2, p. 88-103, 2009.

Jin, S. A. A. Modality effects in Second Life: the mediating role of social presence and the moderating role of product involvement. CyberPsychology E Behavior, v. 12, n. 6, p. 717-721, 2009.

Johnson, R. D. Gender differences in e-learning: communication, social presence and learning outcomes. Journal of Organizational and End User Computing, v. 23, n. 1, p. 79-94, jan./mar. 2011.

KeAR, K. Social presence in online learning communities. In: $7^{\text {th }}$ international conference on networked learning 2010. Aalborg, Denmark: [s.n.]. 2010.

Kehrwald, B. Understanding social presence in text-based online learning environments. Distance Education, v. 29, n. 1, p. 89-106, mai. 2008.

Kıм, J. J. Developing an instrument to measure social presence in distance higher education. British Journal of Educational Technology, v. 42, n. 5, p. 763-777, 2011.

Kıм, J. J.; Kwona, Y.; Сно, D. Investigating factors that influence social presence and learning outcomes in distance higher education. Computers $\&$ Education, v. 57, p. 1512-1520, 2011.

Kreijns, K.; Kirschner, P.A.; Jochems, W. Identifying the pitfalls for social interaction in computer-supported collaborative learning environments: a review of the research. Computers in Human Behavior, v. 19, p. 335-353, 2003.

Lin, G. Y. Social presence questionnaire of online collaborative learning: development and validity. In: Annual Meeting of the Association for Educational Communications and Technology. Chicago, IL: [s.n.]. 2004.

Liu, S. Y.; Gomez, J.; Yen, C. J. Community college online course retention and final grade: predictability of social presence. Journal of Interactive Online Learning, v. 8, n. 2, p. 165-182, jul./set. 2009. 
Lowenthal, P. R. The evolution and influence of Social Presence Theory on online learning. In: KIDD, T. Online education and adult learning: new frontiers for teaching practices. Hershey: IGI Global, 2010.

Mackey, K. M.; Freyberg, D. L. The effect of social presence on affective and cognitive learning in an international engineering course taught via distance learning. Journal of Engineering Education, v. 99, n. 1, p. 23-34, jan. 2010.

Mattar, J. Interatividade e aprendizagem. In: Litto, F. M.; Formiga, M. (Orgs.). Educação a distância: o estado da arte. São Paulo: Pearson Education do Brasil, 2009.

Mehlecke, Q. T. C. Relações dialógicas no ambiente de suporte à educação on-line: um estudo das estratégias no contexto de falantes da língua portuguesa de Portugal.2006. 159f. Tese (Doutorado em Informática na Educação) - Universidade Federal do Rio Grande do Sul (UFRGS). Porto Alegre, 2006.

Morueta, R. T.; Gómez, Á.H.; Gómez,J.I. A. Aprendizaje cooperativo on-line através de foros en un contexto universitario: un análises del discurso y de las redes. Estudios sobre Educación, v. 20, p. 49-71, 2011.

Mueller, D.; Strohmeier, S. Design characteristics of virtual learning environments: an expert study. International Journal of Training and Development, v. 14, n. 3, p. 209-222, 2010. Oncu, S.; CaKir, H. Research in online learning environments: priorities and methodologies. Computers E Education, v. 57, p. 1098-1108, 2011.

Onrubia, J.; Colomina, R.; Engel, A. As comunidades virtuais de aprendizagem baseadas no trabalho em grupo e na aprendizagem colaborativa. In: Coll, C.; Monereo, C. (Orgs.). Psicologia da educaşão virtual: aprender e ensinar com as tecnologias da informação e da comunicação. Porto Alegre: Artmed, 2010.

Primo, A. F. T. Interação mediada pelo computador: a comunicação e a educação a distância segundo uma perspectiva sistêmico-relacional. 2003. 290f. Tese (Doutorado em Informática na Educação) - Universidade Federal do Rio Grande do Sul (UFRGS). Porto Alegre, 2003.

Quintas-Mendes, A.; Morgado, L.; Amante, L. Comunicação mediatizada por computador e educação online: da distância à proximidade. In: Silva, M.; Pesce, L.; Zuin, A. Educação online: cenário, formação e questões. Rio de Janeiro: WAK, 2010.

Richardson, J. C.; Swan, K. Examining social presence in online courses in relation to students' perceived learning and satisfaction. Journal of Asynchronous Learning Network, v. 7, n. 1, p. 68-88, fev. 2003.

Russo, T.; Benson, S.. Learning with invisible others: perceptions of online presence and their relationship to cognitive and affective learning. Educational Technology $\mathcal{E}^{2}$ Society, v. 8, n. 1, p. 54-62, 2005.

Salvat, B. G.; Silva, J. Metodologías para el análisis de espacios virtuales colaborativos. Revista de Educación a Distancia, v. 16, 2006.

Short, J.; Williams, E.; Christie, B. The social psychology of telecommunication. New York: John Wiley, 1976.

Sung, E.; Mayer, R. E. Five facets of social presence in online distance education. Computers in Human Behavior, v. 28, p. 1738-1747, 2012. 
Tu, C. H. On-line learning migration: from social learning theory to social presence theory in a CMC environment. Journal of Network and Computer Applications, v. 23, p. $27-37,2000$.

. The impacts of text-based CMC on online social presence. The Journal of Interactive Online Learning, v. 1, n. 2, 2002.

Tu, C. H.; McisaAc, M. The relationship of social presence and interactions in online classes. The American Journal of Distance Education, v. 16, n. 3, p. 131-150, 2002.

Vygotsky, L. S. A formação social da mente. 4. ed. São Paulo: Martins Fontes, 1991.

Wallon, H. As origens do caráter na criança. São Paulo: Nova Alexandria, 1995.

Wei, C. -W.; Chen, N. S.; Kinshuk. A model for social presence in online classrooms. Educational Technology Research and Development, v. 60, p. 529-545, 2012.

Woo, Y.; Reeves, T. C. Meaningful interaction in web-based learning: a social constructivist interpretation. Internet and Higher Education, v. 10, p. 15-25, 2007.

Yen, C. J.; Tu, C. H. A multiple-group confirmatry factor analysis of the scores for online social presence: do they measure the same things across cultural groups? Journal Educational Computing Research, v. 44, n. 2, p. 219-242, 2011.

\section{SOBRE OS AUTORES}

Willyans Garcia Coelho é doutorando em psicologia social na Universidade Federal da Paraíba (UFPB). Professor do Instituto Federal de Pernambuco (IFPE).

E-mail:willyans@rh.com.br

Patricia Cabral de Azevedo Restellei Tedesco é doutora em ciência da computação pela University of Leeds Computer Based Learning Unit (Grã-Bretanha). Professora da Universidade Federal de Pernambuco (UFPE). E-mail: pcart@cin.ufpe.br

Recebido em 22 de abril de 2014 Aprovado em 29 de junho de 2015 